\title{
Long Range Magnetic Interaction between Josephson Junctions
}

\section{Grønbech-Jensen, Niels; Samuelsen, Mogens Rugholm}

\section{Published in:}

Physical Review Letters

Link to article, DOI:

10.1103/PhysRevLett.74.170

Publication date:

1995

Document Version

Publisher's PDF, also known as Version of record

Link back to DTU Orbit

Citation (APA):

Grønbech-Jensen, N., \& Samuelsen, M. R. (1995). Long Range Magnetic Interaction between Josephson Junctions. Physical Review Letters, 74(1), 170-173. https://doi.org/10.1103/PhysRevLett.74.170

\section{General rights}

Copyright and moral rights for the publications made accessible in the public portal are retained by the authors and/or other copyright owners and it is a condition of accessing publications that users recognise and abide by the legal requirements associated with these rights.

- Users may download and print one copy of any publication from the public portal for the purpose of private study or research.

- You may not further distribute the material or use it for any profit-making activity or commercial gain

- You may freely distribute the URL identifying the publication in the public portal

If you believe that this document breaches copyright please contact us providing details, and we will remove access to the work immediately and investigate your claim 


\title{
Long Range Magnetic Interaction between Josephson Junctions
}

\author{
Niels Grønbech-Jensen \\ Theoretical Division, Los Alamos National Laboratory, Los Alamos, New Mexico 87545 \\ Mogens R. Samuelsen \\ Physics Department, The Technical University of Denmark, DK-2800 Lyngby, Denmark
}

(Received 8 July 1994)

\begin{abstract}
A new model for magnetic coupling between long Josephson junctions is proposed. The coupling mechanism is a result of the magnetic fields outside the junctions and is consequently effective over long distances between junctions. We give specific expressions for the form and magnitude of the interaction, and we study a few dynamical examples of experimental relevance. It is found that this new coupling manifests itself much like Volkov's coupling through thin superconductors.
\end{abstract}

PACS numbers: 74.50.+r, 03.40.Kf, 85.25.Cp

A long Josephson junction ( $\mathrm{LJJ})$ is typically characterized by its internal properties such as capacitance, surface inductance, Josephson energy, and tunneling of quasiparticles [1]. However, due to the spatial variation of the surface currents in the superconductors defining the junction, magnetic fields are created outside the junction. If two or more LJJ's are in close proximity vicinity, these external magnetic fields will overlap and, hence, cause interaction between the individual junctions (we will refer to this as external magnetic coupling). This idea was the basis for the experimental and theoretical works of Holst et al. [2]. A local interaction model was created in order to explain the experimental results. This model has been able to explain experimental features like phase locking $[3,4]$, power emissions [5], and splitting of the characteristic fluxon velocities [3]. The model of local magnetic interaction turned out to be similar to another coupling mechanism between closely stacked LJJ's, suggested by Ngai [6]. This coupling mechanism, later considered by other authors [7-9] and finally parametrized by Volkov [10], is concerned with interactions of currents and magnetic fields in thin superconductors between junctions (we will refer to this as internal magnetic coupling).

In this paper we will parametrize the external magnetic coupling. We will see that the external coupling in general must be modeled as a nonlocal interaction, but in some limiting cases we can regard the coupling as local. New effects are predicted as a result of the nonlocal interaction. Since the external coupling always accompanies the internal (the opposite is not the case), we discuss the importances of the external coupling relative to the internal.

A single overlap LJJ is modeled by [1]

$$
\phi_{x x}-\phi_{t t}-\sin \phi=\alpha \phi_{t}-\eta,
$$

where $\phi$ denotes the difference between the phases of the quantum mechanical wave functions defining the junction. The spatial $(x)$ and temporal $(t)$ coordinates are normalized to the Josephson length $\lambda_{J}=\sqrt{\hbar / 2 e d \mu_{0} I_{c}}$ and the inverse plasma frequency $\omega_{p}^{-1}=\sqrt{\hbar \epsilon / 2 e t_{0} I_{c}}$, respec- tively. Here, the permeability is given by $\mu_{0}$, the permittivity by $\epsilon$, and the critical current density is $I_{c}$. The electric and magnetic thicknesses of the junction are given by the insulating layer $t_{0}$ and $d=2 \lambda_{L}+t_{0}, \lambda_{L}$ being the magnetic penetration depth of the superconductors. The bias current density (per length) $\eta$ is normalized to the critical current density $W I_{c}$ of the junction, and voltages $\left(\phi_{t}\right)$ are normalized to $\hbar \omega_{p} / 2 e$. The dissipative parameter $\alpha$, representing tunneling of quasiparticles, is given by $\alpha=G \hbar \omega_{p} / 2 e I_{c}$, where $G$ is the conductance density of the junction in the normal state. Energy is normalized to $H_{0}=I_{c} W \lambda_{J} \hbar / 2 e=W(\hbar / 2 e)^{2} / \mu_{0} d \lambda_{J}$, where the width of the junction is $W$. We assume $W<\lambda_{J}$ in order to consider the dynamics of the junction one dimensional. The spatial derivative $\phi_{x}(x)$ denotes the normalized surface current density in the superconductors at the point $x$. The magnetic flux $d M_{\phi}$ over an interval $d x$ at point $x$ is therefore given by

$$
d M_{\phi}=\frac{\hbar}{2 e} \phi_{x}(x) d x,
$$

where $d M_{\phi}$ is equivalent to a magnetic charge at the edge of the junction.

Let us now consider two adjacent LJJ's, represented by the phase variables, $\phi_{1}\left(x_{1}\right)$ and $\phi_{2}\left(x_{2}\right)$. The $x_{1}$ and $x_{2}$ axes are parallel as illustrated in Fig. 1, where two relevant geometries are shown. Because of the external magnetic fields discussed above, these junctions will experience a normalized Coulomb-like interaction energy $\left[d H_{\text {int }}=\right.$ $d M_{\phi_{1}} d M_{\phi_{2}} / 4 \pi \mu_{0} r$, where $r=\sqrt{S^{2}+\lambda_{J}^{2}\left(x_{2}-x_{1}\right)^{2}}$ is the physical distance between the two charge contributions, $S$ being the physical distance between the edges of the junctions],

$$
\begin{aligned}
& h_{\mathrm{int}}=\iint g\left(x_{1}-x_{2}\right) \phi_{1, x_{1}} \phi_{2, x_{2}} d x_{1} d x_{2}, \\
& g(z)=\frac{\Delta_{0}}{\sqrt{1+z^{2} / s^{2}}}, \quad \Delta_{0}=\frac{N}{4 \pi} \frac{d \lambda_{J}}{W S},
\end{aligned}
$$

where $s=S / \lambda_{J}$, and $\lambda_{L} \ll S$ is formally assumed when considering $d M_{\phi}$ a point charge. The geometrical pre- 


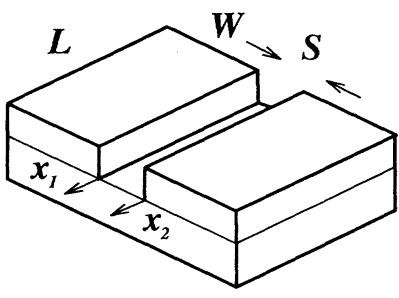

(a)

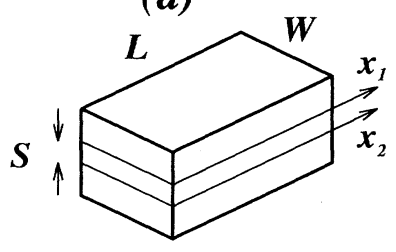

(b)

FIG. 1. Schematic diagrams of magnetically coupled long Josephson junctions. Adjacent geometry proposed in Ref. [2] (a). Stacked geometry of $[6,7,10]$ (b).

factor $N$, which is 1 if no superconductors are present, is estimated as follows. First of all we assume idealized semi-infinite geometry. Under this assumption a factor of $2^{2}$ is contributed to $N$ from concentrating the fields on one side of the semi-infinite superconductors. A factor of $\frac{1}{2}$ is contributed from the fact that the energy density should be integrated over the space on one side of the superconductors only. Finally a factor of 2 comes from the "mirror" screening currents in the superconductors. We therefore estimate $N$ to be 4 for the geometry shown in Fig. 1(a). For the stacked geometry shown in Fig. 1(b), an additional factor of 2 comes from the two equivalent sides of the superconductors. We therefore estimate $N$ to be 8 for the stacked geometry. We note that these estimates are correct only for perfect (semi-infinite) geometries. For real, finite size, geometries we should expect the prefactor $N$ to deviate from 4 or 8 .

Following Ref. [11] the above magnetic interaction can be included in the dynamical field equations in the following form:

$$
\begin{aligned}
\phi_{i, x_{i} x_{i}}-\phi_{i, t t}-\sin \phi_{i}= & \alpha \phi_{i, t}-\eta_{i} \\
& -\int g_{x_{i}}\left(x_{i}-x_{j}\right) \phi_{j, x_{j}} d x_{j},
\end{aligned}
$$

where $i$ and $j(=1,2$ and $i \neq j$ ) denote the two junctions and their variables.

Defining the total normalized energy $h$ by

$$
\begin{gathered}
h=h_{\phi_{1}}+h_{\phi_{2}}+h_{\mathrm{int}}, \\
h_{\phi_{i}}=\int\left[\frac{1}{2} \phi_{i, x_{i}}^{2}+\frac{1}{2} \phi_{i, t}^{2}+1-\cos \phi_{i}\right] d x_{i} .
\end{gathered}
$$

Assuming that the system has the normalized length $l=$ $L / \lambda_{J}$ and using Eq. (5), we obtain the time derivative of the total energy as

$$
\begin{aligned}
\dot{h}= & \left.\sum_{i=1}^{2}\left[\phi_{i, x_{i}} \phi_{i, t}+\phi_{i, t} \int g\left(x_{i}-x_{j}\right) \phi_{j, x_{j}} d x_{j}\right]\right|_{x_{i}=-l / 2} ^{x_{i}=l / 2} \\
& -\sum_{i=1}^{2}\left\{\alpha \int \phi_{i, t}^{2} d x_{i}-\eta_{i} \int \phi_{i, t} d x_{i}\right\}
\end{aligned}
$$

From this expression we see that, for $\alpha=\eta_{1}=\eta_{2}=0$, the energy is conserved if we choose

$$
\phi_{i, x_{i}}( \pm l / 2)=-\int_{-l / 2}^{l / 2} g\left( \pm l / 2-x_{j}\right) \phi_{j, x_{j}} d x_{j} .
$$

This condition is consistent with the usual boundary conditions for LJJ's, since the right-hand side of Eq. (9) is the magnetic field at the boundary originating from the other junction. Notice that for $l \rightarrow \infty$ or for periodic boundaries, $\phi_{i}(l / 2)=\phi_{i}(-l / 2)-2 \pi n_{i}$, the energy is automatically conserved when $\alpha=\eta_{1}=\eta_{2}=0$.

Let us study the small amplitude limit of the unperturbed coupled system Eq. (5),

$$
\phi_{i, x_{i} x_{i}}-\phi_{i, t t}-\phi_{i}=-\int g_{x_{i}}\left(x_{i}-x_{j}\right) \phi_{j, x_{j}} d x_{j} .
$$

Evaluating the spatial Fourier transform of these equations, adding and subtracting, yields

$$
\left(\omega^{2}-k^{2}-1\right)\left(\phi_{1, k} \pm \phi_{2, k}\right)=\mp k^{2} G(k)\left(\phi_{1, k} \pm \phi_{2, k}\right),
$$

where $G(k)$ is the Fourier transform of $g(z)$ : $G(k)=$ $2 s \Delta_{0} K_{0}(s|k|) . \quad K_{n}(z)$ is the modified Bessel function of second kind and order $n$. Clearly two dispersion relations are identified from Eq. (11),

$$
\omega(k)=\sqrt{1+[1 \mp G(k)] k^{2}},
$$

and from this we can evaluate characteristic phase velocities, $c_{\mathrm{ph}}$, and group velocities, $c_{\mathrm{gr}}$ :

$$
\begin{gathered}
c_{\mathrm{ph}}=\sqrt{1+k^{2}[1 \mp G(k)]} /|k|, \\
c_{\mathrm{gr}}=|k| \frac{1 \mp G(k) \mp \frac{1}{2}|k| G^{\prime}(k)}{\sqrt{1+k^{2}[1 \mp G(k)]}} .
\end{gathered}
$$

It is important to realize the similarity between the external coupling and the internal coupling of the stacked geometry discussed in Refs. $[6,10]$. For the internal coupling it was found that the normalized interaction energy is given by

$$
\begin{aligned}
h_{\mathrm{int}}^{(i)} & =\Delta_{1} \iint \delta\left(x_{1}-x_{2}\right) \phi_{1, x_{1}}\left(x_{1}\right) \phi_{2, x_{2}}\left(x_{2}\right) d x_{1} d x_{2}, \\
\Delta_{1} & =e^{-s^{\prime}}, \quad s^{\prime}=S / \lambda_{L}=s \lambda_{J} / \lambda_{L} .
\end{aligned}
$$

This interaction has the same form as Eq. (3) if $g(z)$ is the Dirac delta function $\delta(z)$. A situation where $g(z)$ (of the external coupling) can be regarded as a delta function is when the distance $s$ between the junctions is small [see Eq. (4)]. Because of the similarity between the external and the internal coupling mechanisms for small distance $s$, we expect the dynamics of the externally coupled system to exhibit many features seen for the internally (locally) coupled system. 
In order to perform simple numerical simulations we have applied periodic boundary conditions for all the following results. Note that the interaction given in Eq. (5) should be understood in the following way for periodic boundary conditions:

$$
\int g_{x_{i}}(z) \phi_{j, x_{j}} d x_{j} \rightarrow \sum_{k=-\infty}^{\infty} \int_{-l / 2}^{l / 2} g_{x_{i}}(z-k l) \phi_{j, x_{j}} d x_{j}
$$

where $z=x_{i}-x_{j}$. Making direct contact to the extensive studies of stability of bunched states for the coupling mechanism given by $\Delta_{1}$ [4], we study a system with one kink soliton in each junction; i.e., the initial condition for each junction is given in the form

$$
\phi_{i}=4 \tan ^{-1} \exp \left[\sigma \frac{x_{i}-u t}{\sqrt{1-u^{2}}}\right],
$$

where $\sigma= \pm 1$ is the polarity of the kink and $u$ is its velocity. As was demonstrated in Refs. [3,4], this solution deforms when $\phi_{1}=\phi_{2}$ in a system coupled by $\Delta_{1}$, and the asymptotic speed is consequently higher than that of the separated mode $\phi_{1} \neq \phi_{2}$. The bunched unipolar mode, $\phi_{1}=\phi_{2}$, is a high energy state [see Eq. (3)] and therefore unstable in most cases. However, it has been shown to be stable for the internal coupling if the translational velocity exceeds a certain critical value [4]. Therefore, below this threshold the mode $\phi_{1}=\phi_{2}$ is unstable and the high velocity state switches to the low velocity state indicating the separated mode, $\phi_{1} \neq \phi_{2}$. The difference between those two characteristic velocities, as well as the critical velocity for which the bunched $\left(\phi_{1}=\phi_{2}\right)$ mode is stable, indicates the strength of the coupling mechanism $\Delta_{1}$. We note that even if the coupling is too weak to stabilize the bunched $\phi_{1}=\phi_{2}$ mode the system may still exhibit strong phase locking, based on the repulsion between the solitons (see Refs. [2,3] for details).

In Fig. 2 we have shown simulated current-voltage (IV) characteristics $\left(V=u \pi \omega_{p} \hbar / l e\right)$ and group velocities for a system with the following parameters: $l=10, \alpha=$ 0.1 , and $\eta_{1}=\eta_{2}$. Consider a coupling given by the experimental geometry in Ref. [2] [geometry shown in Fig. 1(a)]: $\lambda_{J}=250 \mu \mathrm{m}, \lambda_{L}=90 \mathrm{~nm}, W=20 \mu \mathrm{m}$, and $S=35 \mu \mathrm{m}$. From these values we obtain the coupling parameters: $\Delta_{0}=0.021$ and $s=0.14$ from Eq. (4). The results of the simulations are shown in Fig. 2 as thin solid lines labeled "1." For high bias we find clear evidence of two characteristic velocities as given by Eq. (14) and shown in Fig. 2(b). The high velocity is found for the $\phi_{1}=\phi_{2}$ mode, whereas the other indicates the separated $\phi_{1} \neq \phi_{2}$ mode. A magnification of the $I V$ structure with the two characteristic velocities is shown as an inset in Fig. 2(a). Decreasing the bias for the high speed mode, the system reaches a critical point where the stability of the bunched mode $\phi_{1}=\phi_{2}$ vanishes. Below this point the two solitons will always repel each other and the solitons move according to the separated mode (low speed). This is qualitatively the same phenomenon
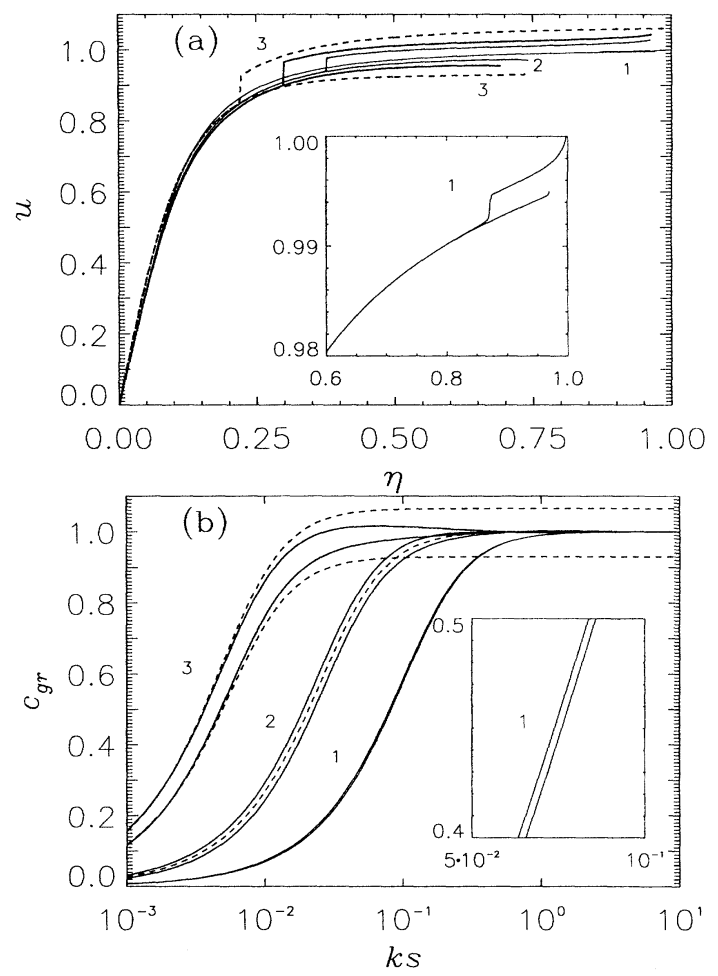

FIG. 2. Normalized $I V$ curves for a coupled system with one soliton in each junction (a). Group velocities calculated from Eq. (14) (b). Parameters are $l=10, \alpha=0.1$, and periodic boundary conditions. Case 1: thin curves represent the external coupling, $\Delta_{0}=0.021$, and $s=0.14$. Case 2: medium solid curves represent the external coupling, $\Delta_{0}=0.318$ and $s=$ 0.036 [dashed curve in (b) represents the group velocities for the corresponding internal coupling, $\left.\Delta_{1} \approx 0.5 \times 10^{-4}\right]$. Case 3 : Thick solid curves represent the external coupling, $\Delta_{0}=1.592$ and $s=0.0072$, and the dashed curves represent the results of the corresponding internal coupling, $\Delta_{1}=0.135$. Inset shows a magnification of case 1 .

as was explained for the internal coupling $\left(\Delta_{1}\right)$ [4], and the fact that we can identify the two characteristic velocities implies that the effective interaction between the junction is sufficiently strong to produce substantial phase locking-even in this relatively weakly coupled system, as was also observed experimentally [2]. We note that there is no internal coupling for this geometry.

If we consider the stacked geometry, shown in Fig. 1(b), typical experimental parameters can be found in Refs. [12,13]: $\lambda_{J}=25 \mu \mathrm{m}, \quad \lambda_{L}=90 \mathrm{~nm}$, and $W=10 \mu \mathrm{m}$. In Fig. 2 we have shown results for a system with $S=10 \lambda_{L}$ as the medium thickness curves labeled " 2 ." The internal coupling parameter is $\Delta_{1} \approx 0.5 \times 10^{-4}$ and therefore insignificant [see Eq. (15)]. The external coupling, in this case given by the parameters, $\Delta_{0}=0.318$ and $s=0.036$, demonstrates strong interaction with two well-separated characteristic velocities, which are also seen from the group velocities, 
shown in Fig. 2(b). The dashed curve, labeled " 2 " in Fig. 2(b), represents the two (indistinguishable) group velocities for the internal coupling, $\Delta_{1} \approx 0.5 \times 10^{-4}$.

Finally the situation for $S=2 \lambda_{L}$ is shown as thick curves, labeled " 3 ." The external coupling is given by $\Delta_{0}=1.592$ and $s=0.0072$, whereas the internal coupling is given by $\Delta_{1}=0.135$. For this geometry the internal and external coupling mechanisms result in almost the same $I V$ characteristics (the results from the internal coupling are shown as dashed thick curves in Fig. 2). If the distance $S$ was chosen even smaller, we would observe the internal coupling as the dominant of the two.

From the above simulations we see that the external coupling mechanism, parametrized in this paper as a twoparameter model with nonlocal interaction, can produce strong interaction over very long distances between two junctions. We have demonstrated that the experimental geometry reported by Holst et al. [2] presents magnetic interaction strong enough to produce substantial phase locking between fluxons. We have also demonstrated the close similarity between the external and internal coupling mechanisms by observing the two characteristic velocities in the coupled system. In fact, the two coupling mechanisms (the internal and the external) manifest themselves in much the same way, except for their magnitudes.

We note that experiments designed to observe phase locking between LJJ's coupled through, e.g., an external cavity, may have unintentionally included the magnetic coupling discussed in this paper. One example of such an experimental geometry is presented in Ref. [14], where the geometrical parameters lead to an estimated magnetic coupling of $\Delta_{0}=0.0015$ and $s=0.44$. The effect of the magnetic coupling is in this case insignificant compared to the coupling through the external linear resonator. However, the magnetic coupling can nevertheless give rise to phase locking between fluxon motion in individual junctions.

We conclude that this new magnetic interaction may be an important, desirable or undesirable, coupling mechanism between LJJ's fabricated in an array. Many experiments are reported, e.g., [2,12-15], on phase locking between LJJ's, where the external magnetic coupling may have played a role in the dynamics. It is therefore important to verify this coupling mechanism experimentally by fabricating, e.g., a stacked structure with parameters similar to what is shown in Fig. 2 (case 2). Such an experiment, where the internal coupling mechanism has no effect, should demonstrate strong coupling with clearly separated characteristic velocities [see Fig. 2 (case 2)] predicted by the external magnetic coupling.

We thank David Cai and Peter Lomdahl for carefully reading the manuscript. Parts of this work were performed under the auspices of the U.S. Department of Energy.

[1] See, e.g., A. Barone and G. Paterno, Physics and Applications of the Josephson Effect (John Wiley \& Sons, New York, 1982).

[2] T. Holst, J. B. Hansen, N. Grønbech-Jensen, and J.A. Blackburn, Phys. Rev. B 42, 127 (1990); IEEE Trans. Magn. 27, 2704 (1991).

[3] N. Grønbech-Jensen, M. R. Samuelsen, P. S. Lomdahl, and J. A. Blackburn, Phys. Rev. B 42, 3976 (1990).

[4] Niels Grønbech-Jensen, David Cai, and Mogens R. Samuelsen, Phys. Rev. B 48, 16160 (1993); Niels Grønbech-Jensen, David Cai, A. R. Bishop, A. W. C. Lau, and Peter S. Lomdahl, Phys. Rev. B 50, 6352 (1994).

[5] Niels Grønbech-Jensen and James A. Blackburn, Phys. Rev. Lett. 70, 1251 (1993); J. Appl. Phys. 74, 6432 (1993).

[6] K. L. Ngai, Phys. Rev. 182, 555 (1969).

[7] M. B. Mineev, G.S. Mkrtchyan, and V.V. Schmidt, J. Low Temp. Phys. 45, 497 (1981).

[8] Yu. S. Kivshar and B. A. Malomed, Phys. Rev. B 37, 9325 (1988).

[9] Yu. S. Kivshar and B. A. Malomed, Rev. Mod. Phys. 61, 763 (1989).

[10] A. F. Volkov, Pis'ma Zh. Eksp. Teor. Fiz. 45, 299 (1987) [JETP Lett. 45, 376 (1987)].

[11] N. Grønbech-Jensen, P.S. Lomdahl, and M.R. Samuelsen, Phys. Lett. A 154, 14 (1991); Niels Grønbech-Jensen, Phys. Rev. B 45, 7315 (1992).

[12] A. V. Ustinov, H. Kohlstedt, and C. Heiden, Appl. Phys. Lett. 65, 1457 (1994).

[13] P. Barbara, A. Ustinov, and G. Costabile, Phys. Lett. A 191, 443 (1994).

[14] A. Davidson, N. Grønbech-Jensen, and N.F. Pedersen, IEEE Trans. Magn. MAG-27, 3347 (1991).

[15] A. V. Ustinov, H. Kohlstedt, M. Cirillo, N. F. Pedersen, G. Hallmanns, and C. Heiden, Phys. Rev. B 48, 10614 (1993). 